\title{
Spatial Distributions of Female Child Marriage and Associated Factors in Ethiopia: Evidence from Demographic and Health Survey
}

Kassahun Tiruaynet Gessesse ( $\sim$ kgesstiru23@gmail.com )

Debre Berhan University

Kindie Fentahun Muchie

Bahir Dar University

Research article

Keywords: Child marriage, female, spatial distribution, associated factors, 20-24 years, Ethiopia

Posted Date: August 6th, 2020

DOI: https://doi.org/10.21203/rs.3.rs-39650/v1

License: (c) (i) This work is licensed under a Creative Commons Attribution 4.0 International License.

Read Full License 


\section{Spatial distributions of female child marriage and associated factors in Ethiopia: evidence from demographic and health survey}

Kassahun Tiruaynet Gessesse ${ }^{1 *}$ and Kindie Fentahun Muchie ${ }^{2}$

${ }^{1}$ Senior Lecturer in Gender and Development Studies, College of Social Science and Humanities, Debre Berhan University, Debre Berhan, Ethiopia

Email: kgesstiru23@gmail.com

${ }^{2}$ Department of Epidemiology and Biostatistics, School of Public Health, College of Medicine and Health Sciences, Bahir Dar University, Bahir Dar, Ethiopia

Email: mkindief@gmail.com

*Correspondence 


\begin{abstract}
Background: In Ethiopia, around million girls were exposed to the risk of child marriage in the country according to previous study report. But this report was not clearly identified and documented the potential local clusters of prevalence of child marriage and associated factors in the country.
\end{abstract}

Objective: This study was aimed to assess spatial distributions of female child marriage and associated factors in Ethiopia using the 2016 Ethiopian demographic and health survey data.

Methods: The study was conducted based on the 2016 Ethiopian demographic and health survey. Data analyzed were taken from 2903 women aged 20-24 years. Local Anselin Moran's I was used to identify potential local clusters of high prevalence of child marriage. Generalized estimating equations for binary outcome was used to identify associated factors of child marriage. Adjusted odds ratio (AOR) with its respective 95\% confidence interval was reported to show the strength of association.

Results: The finding of this study showed that prevalence of child marriage in Ethiopia was $40.2 \%$ [95\%CI: 38.4, 42.0]. In this study also, potential local clusters of high prevalence of child marriage were identified. Local region and educational status were determinant factors of child marriage in the country at $5 \%$ level of significance.

Conclusions: In this study, local region and educational status were determinant factors of child marriage in the country. To address the problems of child marriage concerned government bodies in collaboration with partners should provide massive awareness creation about child marriage and its effects in the country for the local communities.

Key words: Child marriage, female, spatial distribution, associated factors, 20-24 years, Ethiopia 


\section{Background}

Child marriage is any union of couples before the age of 18 [1]. It is a violation of children and women rights in particular and human rights in general [2]. Although child marriage affects both sexes, girls are more victims [3, 4]. It exposes girls to risk of early pregnancy, HIV/AIDS, cervical cancer and obstetric fistulas [5]. It has also the potential adverse consequences for women's mental, emotional and physical development and well-being [6-8].

Despite majority of countries in the world adopted different International Conventions and Agreements and made domestic laws regarding age at marriage, their implementations are hardly on the ground in developing regions [2, 9, 10]. Sub-Saharan Africa and South Asia were good examples of developing regions that child marriage is highly practiced at this contemporary period $[3,9,11]$.

In Ethiopia, even though the government outlined 18 years old as a legal age of marriage, around million girls were exposed to the risk of early marriage in the country [10]. The prevalence of child marriage in Ethiopia was reported as $41 \%$ with an average age at first marriage 16.5 years in 2011 [12].

A few previous studies had been conducted on child marriage and its associated factors in Amhara region, Ethiopia. For instance, earlier studies conducted in Sinan District, South Wollo and East Gojjam Zones reports indicated that education, residence [6,13], media exposure[6] and wealth status[13] were associated factors of child marriage. However, these studies were small coverage area in Amhara region. In addition, prior studies were not documented the potential local clusters of prevalence of child marriage and associated factors in Ethiopia in general and different local regions in particular using evidence from 2016 Ethiopian demographic and health survey till then.

Therefore, this study was aimed to assess spatial distributions of female child marriage and associated factors in Ethiopia using the 2016 Ethiopian demographic and health survey data. To achieve target of elimination of prevalence of child marriage in the country this research is used to the policy makers, partners and concerned government bodies to formulate appropriate strategies and interventions programme. 


\section{Methods}

\section{Study setting}

The 2016 Ethiopian Demographic and Health Survey (EDHS) was conducted in nine regional states of Ethiopia and two city administrations. These are: Tigray, Afar, Amhara, Oromia, Somali, Benishangul-Gumuz, Southern Nations Nationalities and Peoples (SNNP), Gambela Harari, Addis Ababa and Dire Dawa. Ethiopia is one of the Sub-Saharan African countries found in the Horn of Africa with a population of 73.5 million according to 2007 national housing and population census [14]. It is one of the countries which have the highest burden of child marriage in the world. In Ethiopia also, child marriage involves either one or two spouses being children and may take place under civil, religious or customary laws with or without formal registration.

\section{Sample and sampling techniques}

The sample for the survey was designed to represent national, urban-rural, and regional estimates of health and demographic outcomes. The EDHS 2016 samples were also selected using a stratified and two-stage cluster sampling methods. Sketch maps were drawn for each of the clusters, and all conventional households were listed. A total of 15,683 women in the age group 15-49 years were interviewed with a response rate of 95\%. Among those women interviewed, 2903 were aged 20-24 years [14]. However, this study used sample for data analyzed was taken from 2903 women aged 20-24 years.

\section{Data extraction, processing and analysis}

The 2016 EDHS survey data sets and the Global Positioning System (GPS) points were downloaded and processed with permission from the Measure DHS (http://www.dhsprogram.com). Then, social determinants and child marriage prevalence indicator variables were extracted from female data sets. ArcGIS version 10.1 was used for spatial analysis and Stata version 14.0 for the remaining analyses. After understanding the detailed data sets and coding, further data recoding was carried out. Further, the GPS points were merged with the prevalence of child marriage in each EDHS study clusters. The prevalence of child marriage was exported into ArcGIS to visualize clusters of hot and cold spots.

Descriptive statistics were used to determine the prevalence of child marriage across social determinant variables. Spatial analysis was applied to detect geographic variation of prevalence

of child marriage among EDHS clusters. Geographic variation of significant high prevalence or 
low prevalence of child marriage were computed for each cluster using the Moran's I statistic. Maps to show the distribution and variations of child marriage throughout the country were constructed. Further, interpolation was used to supplement Moran's I statistic.

Generalized estimating equations (GEE) for binomial family were used to determine associated factors of child marriage. GEE adjusts the standard errors by accounting clustered observations. Accordingly, the bi-variable GEE of factors on child marriage were fitted. All variables with pvalue $\leq 0.25$ in the bi-variable were fitted in multivariable GEE. Both crude odds ratio (COR) and adjusted odds ratio (AOR) with the corresponding 95\% confidence intervals $(95 \% \mathrm{CI})$ were reported to show the strength of association.

\section{Results}

From a total of 2903 women interviewed, $41.5 \%, 39.1 \%$ and $18 \%$ of respondents were Orthodox Christian, Muslim and Protestant respectively (Table 1). The study also shows majority (62.3\%) of respondents were living in rural areas. Concerning marital status, about $61.4 \%$ of respondents were cohabiting with partners. However, $38.7 \%$ of respondents were non-cohabiting with partners. Regarding educational status, the study also shows 37.1\%, $19.4 \%$ and $14.6 \%$ of respondents were at primary, secondary and higher levels respectively, while the remaining 28.9 $\%$ of respondents were uneducated. In addition, majority $(52.8 \%)$ of respondents had high wealth status.

Table 1 Socio-economic and demographic characteristics of women aged 20-24 years in Ethiopia, 2016-DHS (n=2903)

\begin{tabular}{lll}
\hline Variables & Frequency & Percent \\
\cline { 1 - 2 } Religion & 1204 & \\
Orthodox & 523 & 41.5 \\
Protestant & 1134 & 18.0 \\
Muslim & 42 & 39.1 \\
Other(catholic, traditional and other) & & 1.4 \\
Marital status & 1783 & \\
Cohabiting & 1120 & 61.4 \\
Non-cohabiting ${ }^{@}$ & & 38.6 \\
Residence & & \\
\hline
\end{tabular}




\begin{tabular}{lll}
\hline Rural & 1809 & 62.3 \\
Urban & 1094 & 37.7 \\
Educational level & & \\
Uneducated & 838 & 28.9 \\
Primary & 1077 & 37.1 \\
Secondary & 563 & 19.4 \\
Higher & 425 & 14.6 \\
Wealth status & & \\
Low & 1046 & 36.0 \\
Medium & 329 & 11.3 \\
High & 1528 & 52.7 \\
\hline
\end{tabular}

${ }^{(}$-Married/living together; ${ }^{\circledR @}$-widowed/divorced/single

Out of the total of 2903 interviewed women, $40.2 \%$ (95\% CI: 38.4-42.0) of respondents experienced child marriage in Ethiopia (Table 2). The average age at marriage was 15.02 (s.d=1.65) years, and the median age was 15 (IQR: 14-16) years among those who married. The average age at first birth was $17.2(\mathrm{~s} . \mathrm{d}=1.98)$ years, and the median age at first birth was also 17(IQR: 16-18). About $60.5 \%$ of respondents, among who married in their childhood, were not attending school whereas $39.5 \%$ of respondents were attending school before their first marriage. In this study also, majority (68.3\%) of those who were attending school did not continue to attend school after their marriage. Furthermore, among those married in their childhood, 634 (54.3\%), $501(42.9 \%)$ and $21(1.8 \%)$ of respondents said that their first marriage decided by parents, themselves and relatives respectively.

Table 2 Child marriage, marriage arrangement and attending school among women aged 20-24 years in Ethiopia, 2016-DHS ( $\mathrm{n}=2903$ )

Variable

Frequency Percent

\section{Child marriage}

No

1,736

59.8

Yes

1,167

40.2

Total

2,903

100.0 


\section{Who decided on the first marriage?}

$\begin{array}{ll}\text { Parents } & 634\end{array}$

54.3

Herself

501

42.9

Other family/ relatives

21

1.8

Others

11

0.9

Total

1,167

100.0

Attending school before first marriage?

No

706

60.5

Yes

461

39.5

Total

1,167

100.0

Stopped attending school after first marriage?

No

68.3

Yes

146

31.7

Total

461

100.0

\section{Reason to stop attending school after first marriage?}

Too busy with family life

205

65.1

Husband did not want me to go

70

22.2

Graduated from school

8

2.5

Other

32

10.2

Total

315

100.0

The result of global pattern analysis showed that there was apparent clustering of child marriage in Ethiopia (Moran's I=0.35, z-score $=11.68$, p-value $<0.0001$ ). Hence, from the Anselin's Local 
Index (Figure 1), this study identified local geographical variability of prevalence of child marriage at community level in Ethiopia which was supported by the result of interpolation (Figure 2).

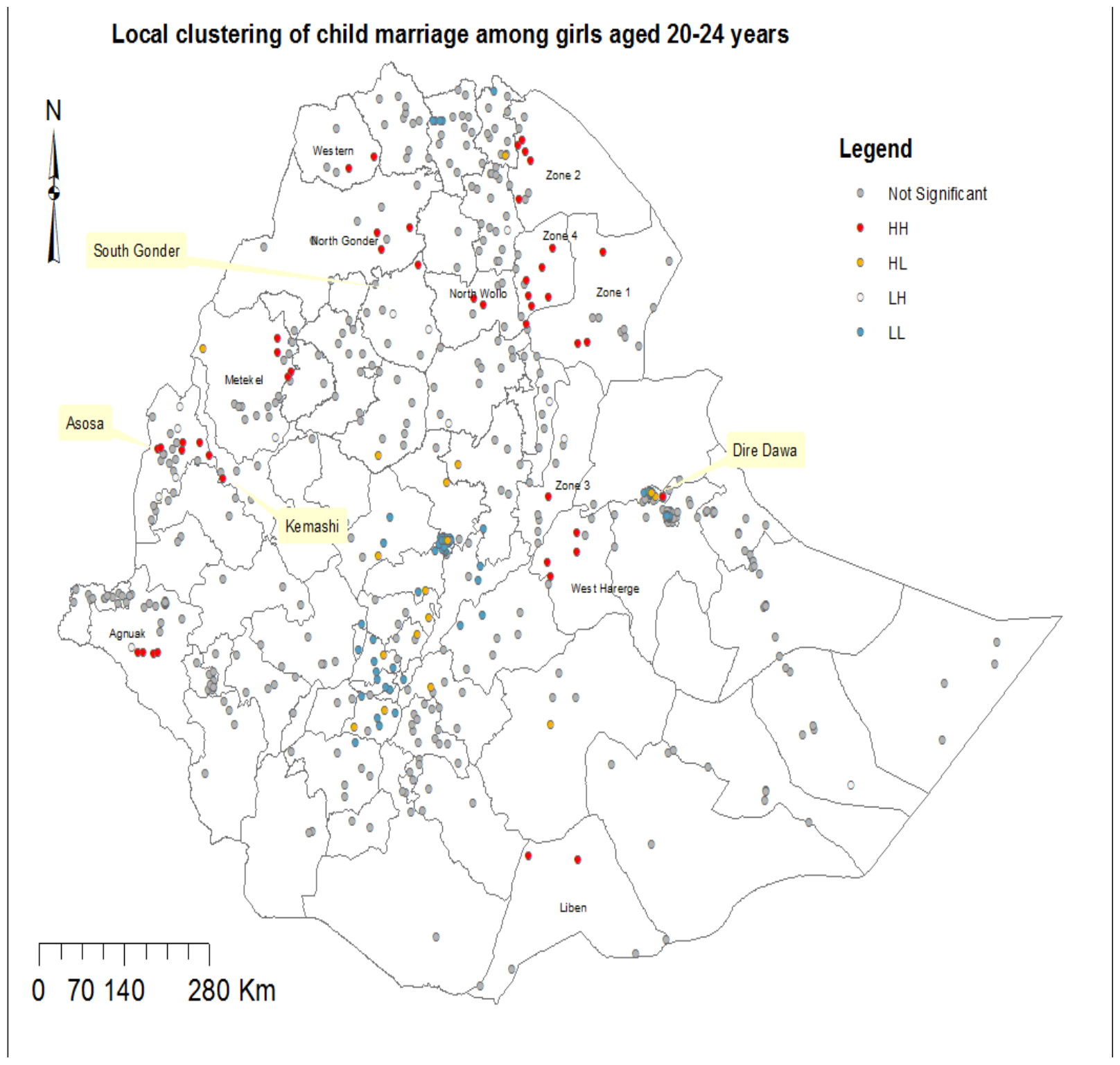

Figure 1 Local clustering of child marriage among girls aged 20-24 years in Ethiopia, 2016 


\section{Percent of child marriage}

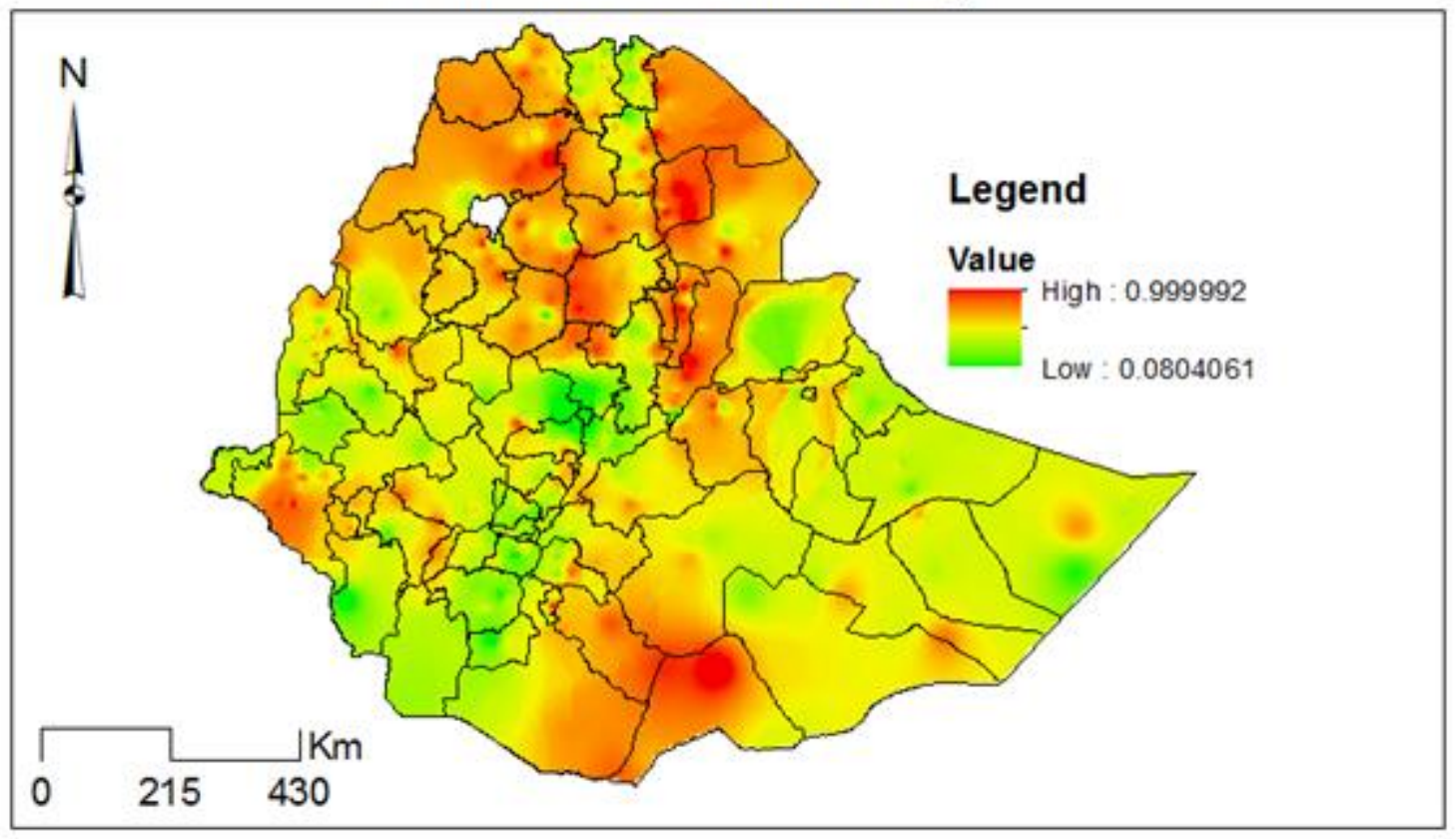

Figure 2 Interpolation of prevalence of child marriage in Ethiopia, 2016

Accordingly, this study identified different local clusters which were detected by high prevalence of child marriage within country. These were: all zones of Afar region; all zones of Amhara region, except Awi zone; three zones in Oromia region (such as Guji, West Harerge, and North Shewa zones); three zones in Tigray region (such as western Tigray, Southern Tigray and Northwestern Tigray); Liben zone in Somali region; and Agnuak zone in Gambela region. In contrast, a study showed that relatively lowest child marriage rates were predicted in Addis Ababa Administration City.

After adjusting for different confounding variables, local region and educational status were significantly associated with child marriage (Table 3). Compared to women living in Addis Ababa Administration City, those living in Gambela [AOR=7.04; 95\%CI=3.79-13.08], Benishangul $[\mathrm{AOR}=6.09 ; 95 \% \mathrm{CI}=3.31-11.21]$, Afar $[\mathrm{AOR}=5.50 ; 95 \% \mathrm{CI}=2.96-10.22]$, Tigray $[\mathrm{AOR}=4.44 ; \quad 95 \% \mathrm{CI}=2.49-7.94], \quad$ Amhara $\quad[\mathrm{AOR}=4.22 ; \quad 95 \% \mathrm{CI}=2.33-7.63]$, Harari [AOR=4.19;95\%CI=2.29-7.65], Oromia $[\mathrm{AOR}=3.44 ; 95 \% \mathrm{CI}=1.93-6.15]$, Somali $[\mathrm{AOR}=2.86$; 95\%CI = 1.56-5.26], Dire Dawa $[\mathrm{AOR}=2.63 ; 95 \% \mathrm{CI}=1.43-4.84]$, and SNNP [AOR=1.96; $95 \% \mathrm{CI}=1.07-3.61]$ had increased probability of child marriage. 
The binary logistic regression also revealed that the odds of child marriage was 1.49 [AOR=2.49; 95\% $\mathrm{CI}=1.60-3.86]$ times higher among women with secondary education, 6.75 [AOR= 7.75; 95\% CI=5.10-11.77] times higher among women with primary education, and 12.46 $[\mathrm{AOR}=13.46 ; 95 \% \mathrm{CI}=8.60-21.07]$ times higher among women with non-educated as compared to women with higher education.

Table 3 Factors associated with child marriage among women aged 20-24 years age in Ethiopia, $(n=2903)$

\begin{tabular}{|c|c|c|c|c|}
\hline \multirow[t]{2}{*}{ Variables } & \multicolumn{2}{|c|}{ Child marriage } & \multirow[t]{2}{*}{ COR $[95 \% \mathrm{CI}]$} & \multirow[t]{2}{*}{$\operatorname{AOR}[95 \% \mathrm{CI}]$} \\
\hline & No $(\%)$ & Yes $(\%)$ & & \\
\hline \multicolumn{5}{|l|}{ Region } \\
\hline Addis Ababa $(\mathrm{RF})^{\mathrm{a}}$ & $334(92.3)$ & $28(7.7)$ & & \\
\hline Tigray & $186(58.7)$ & $131(41.3)$ & $7.58[4.26,13.47]$ & $4.44[2.49,7.94] *$ \\
\hline Afar & $76(32.5)$ & $158(67.5)$ & $23.54[12.87,43.04]$ & $5.50[2.96,10.22] *$ \\
\hline Amhara & $153(55.2)$ & $124(44.8)$ & $8.85[5.00,15.75]$ & $4.22[2.33,7.63] *$ \\
\hline Oromia & $171(52.4)$ & $155(47.6)$ & $10.14[5.75,17.9]$ & $3.44[1.93,6.15] *$ \\
\hline Somali & $120(47.2)$ & $134(52.8)$ & $13.04[7.30,23.31]$ & $2.86[1.56,5.26] *$ \\
\hline Benishangul & $103(49.1)$ & $107(50.9)$ & $12.20[6.71,22.21]$ & $6.09[3.31,11.21] *$ \\
\hline SNNPR & $225(69.9)$ & $97(30.1)$ & $5.31[2.98,9.47]$ & $1.96[1.07,3.61] *$ \\
\hline Gambela & $106(52.7)$ & $95(47.3)$ & $10.30[5.62,18.87]$ & $7.04[3.79,13.08] *$ \\
\hline Harari & $116(59.2)$ & $80(40.8)$ & $7.76[4.23,14.24]$ & $4.19[2.29,7.65] *$ \\
\hline Dire Dawa & $146(71.6)$ & $58(28.4)$ & $4.87[2.62,9.05]$ & $2.63[1.43,4.84] *$ \\
\hline
\end{tabular}


Media exposure

Yes

$814(75.7) \quad 259(24.1) \quad 0.47[0.40,0.56] \quad 1.01[0.81, \quad 1.7]$

No $(\mathrm{RF})^{\mathrm{a}}$

922(50.4) 908(49.6)

\section{Currently working}

Yes

$914(67.4)$

$443(32.6)$

$0.64[0.56,0.75]$

$0.77[0.64, \quad 0.92]$

No (RF) ${ }^{\mathrm{a}}$

822(53.2) 724(46.8)

\section{Residence}

Urban (RF) ${ }^{\mathrm{a}}$

864(79.0) 230(21.0)

Rural

872(48.2) 937(51.8)

\section{Religion}

Protestant $(\mathrm{RF})^{\mathrm{a}}$

334(63.9) $189(36.1)$

Orthodox

$841(69.8) \quad 363(30.2)$

$0.80[0.63,1.02]$

$0.95[0.67, \quad 1.33]$

Muslim

$542(47.8)$

$592(52.2)$

$1.75[1.36,2.25]$

$1.17[0.83, \quad 1.65]$

Other

$19(45.2)$

$23(54.8)$

$1.83[0.96,3.50]$

$1.55[0.75,3.19]$

\section{Educational status}

Uneducated

Primary

Secondary

Higher (RF) ${ }^{\text {a }}$

Wealth status
$295(35.2) \quad 543(64.8) \quad 16.61[11.41,24.17] \quad 13.46[8.60,21.07] *$

$594(55.2) \quad 483(44.8) \quad 7.88[5.50,11.28] \quad 7.75[5.10,11.77] *$

$452(80.3) \quad 111(19.7) \quad 2.60[1.77,3.81] \quad 2.49[1.60,3.86] *$

395(92.9) 30(7.1) 


\begin{tabular}{|c|c|c|c|c|c|}
\hline Low $(\mathrm{RF})^{\mathrm{a}}$ & $439(42.0)$ & $607(58.0)$ & & & \\
\hline Medium & $159(48.3)$ & $170(51.7)$ & $0.80[0.62,1.02]$ & $1.15[0.87$, & $1.52]$ \\
\hline Rich & $1138(74.5)$ & $390(25.5)$ & $0.31[0.26,0.38]$ & $0.86[0.66$ & 1.12] \\
\hline
\end{tabular}

Note: * Significant at $0.05 ;{ }^{\mathbf{a}}$ Reference category

\section{Discussion}

In this study, the prevalence of child marriage in Ethiopia was $40.2 \%$, which means high. The finding of this study also indicated that local region as predictor variable of child marriage in the country, which was strongly significant at 5\% confidence. Factors that contributed to child marriage in local regions were traditional practices, geo-cultural identity, social norms and values of the communities. For instance, previous studies in Ethiopia showed that the rate of prevalence of child marriage in northern Amhara region $(75 \%)$ is three times higher than in Addis Ababa Administration City (26\%) (11). Likewise, earlier study done from Indonesia [15] similar with the present findings that region as a factor of child marriage, implies that the role of religion, ethnic, and other geographically diverse factors.

Educational status was also associated factor of child marriage in this study. The current analysis indicated that uneducated girls were more likely to experience child marriage as compared to those relatively educated. This study was consistent with previous studies done in South Wollo and East Gojjam, Ethiopia [6], in Pakistan [8], in Indonesia [15], in Serbia[16], in Bangladesh $(17,18)$ and Nepal (19). From these across findings we understood that educated girls have more freedom and are better able to exercise their rights than non-educated girls. They were also getting better opportunity to join to school than other non-educated, which are escaping from child marriage.

\section{Conclusion}

In this study, the prevalence of child marriage in Ethiopia was $40.2 \%$. The finding of this study showed that the potential local clusters of high prevalence of child marriage were identified. These are: all zones of Afar region; all zones except Awi/Agew of Amhara region; Guji, West Harerge, and North Shewa zones of Oromia region; three zones in Tigray region (western Tigray, Southern Tigray and North western Tigray); Liben zone of Somali region; and Agnuak 
zone of Gambella region. The findings of this study also showed that local region and educational status were determinant factors of child marriage in the country. So as to solve the problems of child marriage in the study area concerned government bodies in collaboration with partners should provide massive awareness creation about child marriage and its effects in the country for the local communities.

\section{Limitation}

Since detailed information about female child marriage was not collected qualitatively, the qualtative method and analysis were not emplyed in this study.

\section{Abbreviations}

AOR: adjusted odds ratio; CI: confidence intervals; COR: crude odds ratio; EDHS: Ethiopia demographic and health survey; GEE: Generalized estimating equations; GPS: Global Positioning System; and SNNP: Southern Nations Nationalities and Peoples

\section{Acknowledgments}

The authors are grateful to DHS for proving relevant data for this analysis for free.

\section{Author's contributions}

Both authors contributed to designing of the study. KTG and KFM contributed to conceptualizing the idea of the study. KTG defined background of the study and discussed results by supporting with previous study or reviewed the manuscript. KFM defined methods, performed the statistical analysis and interpreted the results. Both authors read and approved the final manuscript.

\section{Funding}

No fund was obtained for this study.

\section{Availability of data and materials}

The data sets analyzed and/or used during the present study available from the corresponding author on reasonable request.

\section{Ethics approval and consent to participate}

Ethical clearance for the 2016 EDHS was provided by the Ethiopian Health and Nutrition Research Institute (EHNRI) Review Board, the National Research Ethics Review Committee (NRERC) at the Ministry of Science and Technology, the Institutional Review Board of ICF 
International, and the CDC. All respondents to the survey provided verbal informed consent; consent for children was obtained through the parents, caregivers or guardians. The data for this study were downloaded and used once the objectives of the analysis was communicated and approved by the Measure DHS.

\section{Consent for publication}

Not applicable

\section{Competing interests}

The authors declare that they have no competing interests.

\section{Author details}

${ }^{1}$ Senior Lecturer in Gender and Development Studies, College of Social Science and Humanities, Debre Berhan University, Debre Berhan, Ethiopia

${ }^{2}$ Department of Epidemiology and Biostatistics, School of Public Health, College of Medicine and Health Sciences, Bahir Dar University, Bahir Dar, Ethiopia

\section{References}

1. Haaland I. Child marriage, well-being and health seeking behavior: a study among married adolescent girls in the Pokot tribe, Karamoja, Uganda. Master thesis in International Community Health, Institute of Health and Society, Faculty of Medicine, University of Oslo.2017. Available at: http://www.duo.uio.no.

2.Rodgers B. Child marriage in Ethiopia and Its associated Human Rights Violations. $U W$ Bothell policy Journal. 2012.

3. Birech J. Child Marriage: A culture Health Phenomenon. International Journal of Humanities and Social Science. 2013;3(17).

4. Pankhurst A, Tiumelissan A, Chuta N. The interplay between community, household and child level influences on trajectories to early marriage in Ethiopia: Evidence from Young Lives. UK: London; 2016.

5. Nour NM. Health consequences of child marriage in Africa. Emerging infectious diseases. 2006;12(11):1644-9.

6. Aserse K, Abebe M. Early Marriage in South Wollo and East Gojjam Zones of Amhara Region, Ethiopia. Humanities and Social Sciences. 2014;2(2):11-6.

7. UNICEF. Surprising trends in child marriage in Ethiopia 2016 [Oct 12, 2018]. Available at : www.unicef.org/Ethiopia.

8. Naveed S, Butt M. Causes and Consequences of Child Marriages in South Asia: Pakistan's perspective. A Research Journal of South Asian Studies. 2015;30(2):161-75.

9. Ahmed T. Child marriage: A Discussion Paper. Bangladesh Journal of Biethics. 2015;6(2):8-14. 
10. Johes N, Emirie G, Tefera B, Presler-Marshall E. Surprising trends in child marriage in Ethiopia. UNICEF, Ethiopia; 2016. Available at: www.unicef.org/Ethiopia.

11. UNICEF. Ending child marriage: progress and prospects. New York: UNICEF; 2014. Available at : http://www.unicef.org/media/files/Child_Marriage_Report_7_17_LR..pdf

12. Central Statistical Agency (CSA) [Ethiopia] and ICF International. Ethiopia Demographic and Health Survey 2011. Addis Ababa, Ethiopia and Calverton, Maryland, USA : CSA and ICF; 2012.

13. Workineh S, Dejenu Kibretb G, Degu G. Determinants of Early Marriage among Female Children in Sinan District, Northwest Ethiopia. Health Science Journal. 2015;9(6).

14. Central Statistical Agency (CSA) [Ethiopia] and ICF. Ethiopia Demographic and Health Survey 2016: Key Indicators Report. Addis Ababa, Ethiopia, and Rockville, Maryland, USA: CSA and ICF; 2016.

15. Rumble L, Peterman A, Irdiana N, Triyana M, Minnick E. An empirical exploration of female child marriage determinants in Indonesia. BMC Public Health. 2018; 18(407).

16. Hotchkiss DR, Godha D, Gage AJ, Cappa C. Risk factors associated with the practice of child marriage among Roma girls in Serbia. BMC international health and human rights. 2016;16(1):6.

17. Jisun TF. Early Marriage of Women: The Case of Bangladesh. World Journal of Social Sciences. 2016;6(2).

18. Nasrin SO, Rahman KM. Factors affecting early marriage and early conception of women: A case of slum areas in Rajshahi City, Bangladesh. International Journal of Sociology and Anthropology. 2012;4(2):54-62.

19. Sekine K, Hodgkin EM. Effective of child marriage on girls' schooling dropout in Nepal: Analysis of data from the multiple indictors cluster survey 2014. PLoS one 2017;12 (17). 


\section{Figures}

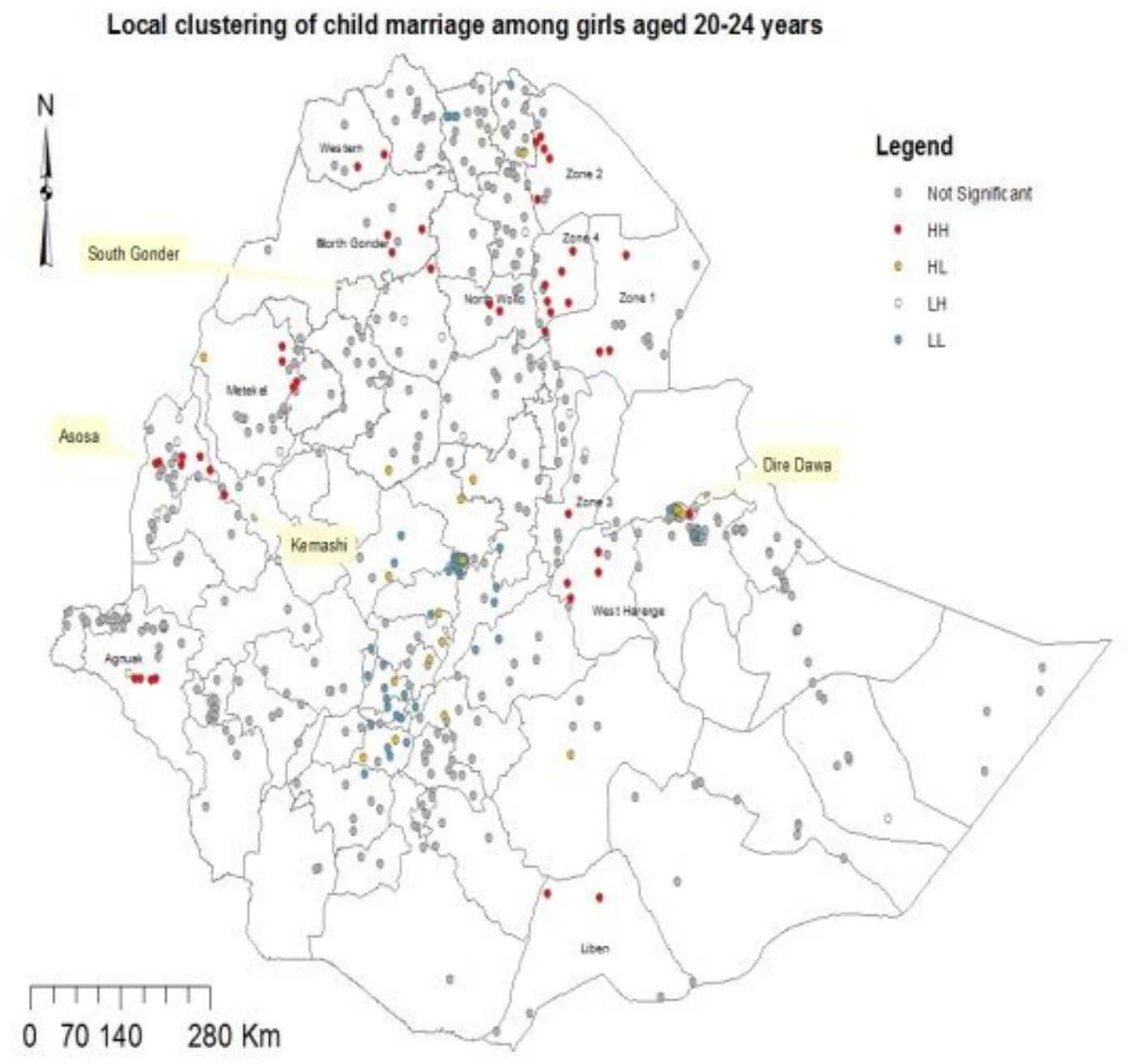

\section{Figure 1}

Local clustering of child marriage among girls aged 20-24 years in Ethiopia, 2016 


\section{Percent of child marriage}

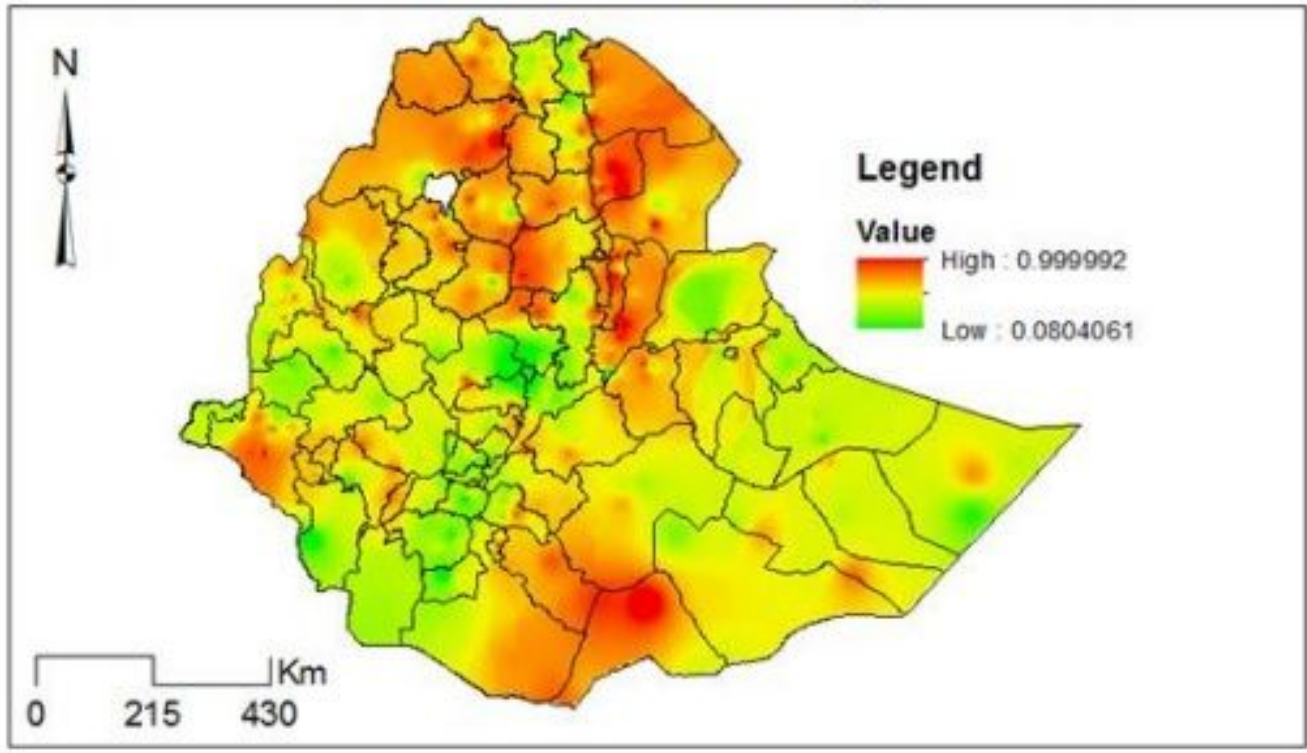

Figure 2

Interpolation of prevalence of child marriage in Ethiopia, 2016 